\title{
An overview of non-local traffic flow models
}

Felisia Angela Chiarello

\begin{abstract}
We give an overview of mathematical traffic flow models with non-local velocity. More precisely, we consider conservation laws with flux functions depending on an integral evaluation of the density of vehicles through a convolution product. We summarize the analytical results recently obtained for this kind of models and we provide some numerical simulations illustrating the behavior of different groups of drivers or vehicles.
\end{abstract}

\section{Introduction}

There are three different important approaches to model traffic flow phenomena: microscopic models, mesoscopic models and macroscopic models. Microscopic models are based on the individual vehicles and their interactions; particular micromodels are cellular automata that prescribe stochastic rules how vehicles behave into cells in which the road is divided; mesoscopic models apply gas-kinetic equations to describe the traffic flow; macroscopic models consider quantities that characterize the collective behaviour of vehicles. In this paper we will consider the framework of macroscopic models. The first macroscopic traffic flow model, based on fluiddynamics equations, has been introduced in transportation literature since the midfifties of last century. It is the Lighthill, Whitham and Richards (LWR) model [27, 29]. The LWR model consists in one scalar equation that expresses the conservation of the number of cars:

$$
\partial_{t} \rho+\partial_{x}(\rho v(\rho))=0,
$$

with $\rho=\rho(t, x)$ representing the mean traffic density, i.e. the number of vehicles per unit length and $v$ denoting the mean velocity. Due to the difficulty to simulate the

Felisia Angela Chiarello

Inria Sophia Antipolis Mediterranée, Université Côte d'Azur, 2004, route des Lucioles- BP 93, 06902 Sophia Antipolis Cedex, France, e-mail: felisia.chiarello@inria.fr 
formation of traffic jams, several approaches have been developed during the years, addressing the need for more sophisticated models. In particular, the classical LWR model does not match the experimental data because it is based on the assumption that the mean traffic velocity is a function of the traffic density, which is not realistic in congested regimes. For these reasons, high order models were developed. In particular, in [28, 31] and [3, 32], the authors considered second order models consisting in a mass conservation equation for the density and an acceleration balance law for the speed. Recently, "non-local" versions of the LWR model have been proposed in $[7,30]$. In this type of models, the speed depends on a weighted mean of the downstream traffic density. As a consequence, the speed is a Lipschitz function with respect to space and time variables, ensuring bounded acceleration and overcoming the limitation of classical macroscopic models that allows for speed discontinuities. Non-local traffic models are intended to describe the behaviour of drivers that adapt their velocity with respect to what happens in front of them. For this reason, the flux function depends on a "downstream" convolution term between the density of vehicles and a kernel function supported on the negative axis $]-\infty, 0]$. As in classical (local) models, the speed is a monotone non-increasing function, because the higher is the density of cars on a road, the lower is their speed. There are general existence and uniqueness results for non-local equations in $[2,21]$ for the scalar case in one-space dimension, in $[17,23]$ for multi-dimensional scalar equations, and in [1] for the multi-dimensional system case. To prove the existence of solutions for these non-local models, there are mainly two different approaches. One is providing suitable compactness estimates on a sequence of approximate solutions constructed through finite volume schemes, as in [1,2]. Another approach relies on characteristics and fixed-point theorems, as proposed in [21, 23]. The first approach [2] requires Kružkov-type entropy conditions to prove the $\mathbf{L}^{\mathbf{1}}$-stability with respect to the initial data through the doubling of variable technique, while in [21,23] the uniqueness of weak solutions is obtained directly from the fixed point theorem. An interesting point is the numerical approximation of the solutions of non-local models. It results challenging due to the high non-linearity of the system and the dependence of the flux function on integral terms, which highly increase the computational cost. There are different ways to numerically integrate non-local conservation laws. A first order Lax-Friedrichs-type numerical scheme is used to approximate the problem and to prove the existence of solutions in $[1,2,6,7,20]$. Another first order numerical scheme is proposed in [19], where an upwind numerical scheme for a class of non-local flux problems is presented. Alternative first order schemes based on the splitting of the non-local equation in two equations, i.e. the Lagrangian and the remap steps are proposed in [14]. In comparison with Lax-Friedrichs scheme and upwind scheme, Lagrangian-Antidiffusive Remap schemes are much less diffusive. Concerning high-order numerical schemes, it is worth citing the papers $[8,13,18]$. In [8], the authors propose discontinuous Galerkin and finite volume WENO schemes to obtain high-order approximations of non-local scalar conservation laws in one space dimension, where the velocity function depends on a weighted mean of the conserved quantity. The discontinuos Galerkin schemes give the best results, but under a very restrictive Courant-Friedrichs-Lewy (CFL) condition. On the contrary, 
finite volume WENO schemes can be implemented on larger time steps. In [13], the authors extend to 1D systems the finite volume WENO schemes proposed in [8]. In [18], central WENO schemes are proposed, which, in contrast with the other high-order schemes for non-local conservation laws, neither require a restrictive CFL condition nor an additional reconstruction step. The paper is organized as follows: Section 2 is devoted to scalar non-local traffic flow models and the corresponding analytical results. A non-local multi-class traffic flow model is recalled in Section 3 and Section 4 presents an upwind numerical scheme, used in Section 5 for numerical tests of the multi-class non-local model with three classes of vehicles.

\section{A class of scalar non-local traffic flow models}

In this section, we recall the class of scalar conservation laws with non-local flux arising in traffic modeling studied in [10]

$$
\partial_{t} \rho+\partial_{x}\left(f(\rho) v\left(\omega_{\eta} * \rho\right)\right)=0, \quad x \in \mathbb{R}, t>0,
$$

where

$$
\omega_{\eta} * \rho(t, x):=\int_{x}^{x+\eta} \omega_{\eta}(y-x) \rho(t, y) d y, \quad \eta>0 .
$$

In (1), (2), we assume the following hypotheses:

$$
\begin{array}{lll} 
& f \in \mathbf{C}^{\mathbf{1}}\left(I ; \mathbb{R}^{+}\right), & I=[a, b] \subseteq \mathbb{R}^{+}, \\
\text {(H) } & v \in \mathbf{C}^{\mathbf{2}}\left(I ; \mathbb{R}^{+}\right) \text {s.t. } & v^{\prime} \leq 0, \\
& \omega_{\eta} \in \mathbf{C}^{\mathbf{1}}\left([0, \eta] ; \mathbb{R}^{+}\right) \text {s.t. } & \omega_{\eta}^{\prime} \leq 0, \\
& \int_{0}^{\eta} \omega_{\eta}(x) d x:=J_{0}, \forall \eta>0, & \lim _{\eta \rightarrow \infty} \omega_{\eta}(0)=0 .
\end{array}
$$

In this model, the function $\rho(t, x)$ represents the density of vehicles on the road and $\eta>0$ is proportional to the look-ahead distance of drivers. Indeed, the aim is to describe the reaction of drivers that adapt their speed with respect to a weighted mean of the downstream traffic density. In particular this class of equations includes the traffic flow models presented in [7, 20,26,30], which are detailed below.

In [30], the authors introduce a traffic flow model based on Arrhenius stochastic microscopic dynamics. Using scaling and limit arguments they obtain a macroscopic description of the microscopic dynamics leading to higher-order dispersive partial differential equations. The dynamics includes interactions with other vehicles ahead. The non-local traffic flow model that derives from this stochastic process is

$$
\left\{\begin{array}{l}
\partial_{t} \rho+\partial_{x}\left(\rho(1-\rho) \exp \left(-\omega_{\eta} * \rho\right)\right)=0 \\
\rho(0, x)=\rho_{0}(x)
\end{array}\right.
$$

where the kernel acts only on the space variable $x$ in this way 


$$
\omega_{\eta} * \rho(t, x)=\int_{x}^{x+\eta} \frac{J_{0}}{\eta} \rho(t, y) d y .
$$

The kernel $\omega_{\eta}$ is an anisotropic short range inter-vehicle interaction potential, $\eta$ is proportional to the look-ahead distance and $J_{0}$ is the interaction strength. Numerical solutions in [25] show that solutions of (3)-(4) allow for shock formations in finite time. In [26], the authors show that the finite time blowup of the solution of (3)-(4) must occur at the level of the first order derivative and the $L^{p}, 1 \leq p \leq \infty$, norms of the solution are finite close to the blowup time. In this way, the results confirm the formation of shock waves for this non-local model.

While pedestrians are likely to react to the presence of people all around them, drivers mostly adapt their velocity to the downstream traffic, assigning a greater importance to closer vehicles. In [7, 20], the authors consider the following mass conservation equation for traffic flow with non-local velocity depending on a mean downstream density:

$$
\partial_{t} \rho(t, x)+\partial_{x}\left(\rho(t, x) v\left(\int_{x}^{x+\eta} \rho(t, y) \omega_{\eta}(y-x) d y\right)\right)=0,
$$

for $t \in \mathbb{R}^{+}$and $x \in \mathbb{R}, \eta>0$. The kernel function $\omega_{\eta} \in \mathbf{C}^{\mathbf{1}}\left([0, \eta] ; \mathbb{R}^{+}\right)$is nonincreasing and the support $\eta$ is proportional to the look-ahead visibility. The mean speed function $v$ is continuous and decreasing. In [5], the authors also study the regularity results for the solutions of this non-local model proving Sobolev estimates and the convergence of approximate solutions solving a viscous non-local equation. The Blandin-Goatin model belongs to the class of equations considered in [21] and in [10]. In [19], the authors propose a general non-local vehicular traffic flow model based on a mean downstream traffic velocity. This is the main difference with the models in [7, 10], where drivers adapt their velocity with respect to a mean downstream traffic density. In particular, the model in [19] allows to capture changes in the velocity function and for this reason, it could be extended to junctions, as in [9].

\subsection{Analytical results for a class of scalar non-local traffic flow models}

Adding an initial condition

$$
\rho(0, x)=\rho_{0}(x), \quad x \in \mathbb{R},
$$

with $\rho_{0} \in \mathrm{BV}(\mathbb{R} ; I)$, entropy weak solutions of the Cauchy problem (1), (5), are intended in the following sense [2, 6, 24].

Definition 1 A function $\rho \in\left(\mathbf{L}^{1} \cap \mathbf{L}^{\infty} \cap \mathrm{BV}\right)\left(\mathbb{R}^{+} \times \mathbb{R} ; I\right)$ is an entropy weak solution of (1), (5), if 


$$
\begin{aligned}
\int_{0}^{+\infty} \int_{\mathbb{R}} & \left\{|\rho-\kappa| \varphi_{t}+\operatorname{sgn}(\rho-\kappa)(f(\rho)-f(\kappa)) v\left(\omega_{\eta} * \rho\right) \varphi_{x}\right. \\
& \left.-\operatorname{sgn}(\rho-\kappa) f(\kappa) v^{\prime}\left(\omega_{\eta} * \rho\right) \partial_{x}\left(\omega_{\eta} * \rho\right) \varphi\right\} d x d t+\int_{\mathbb{R}}\left|\rho_{0}(x)-\kappa\right| \varphi(0, x) d x \geq 0
\end{aligned}
$$

for all $\varphi \in \mathbf{C}_{\mathbf{c}}^{\mathbf{1}}\left(\mathbb{R}^{2} ; \mathbb{R}^{+}\right)$and $\kappa \in \mathbb{R}$.

The following results are obtained in [10], approximating the problem (1)-(5) through a Lax-Friedrichs type numerical scheme and recovering $\mathbf{L}^{\infty}$ and BV estimates for the sequence of approximate solutions. Stability with respect to the initial data is obtained from the entropy condition using the doubling of variable technique.

\section{Theorem 1 (Well-posedness)}

Let hypotheses $(\mathbf{H})$ hold and $\rho_{0} \in B V(\mathbb{R} ; I)$. Then the Cauchy problem (1), (5), admits a unique weak entropy solution $\rho^{\eta}$ in the sense of Definition 1 , such that

$$
\min _{\mathbb{R}}\left\{\rho_{0}\right\} \leq \rho^{\eta}(t, x) \leq \max _{\mathbb{R}}\left\{\rho_{0}\right\}, \quad \text { for a.e. } x \in \mathbb{R}, t>0 .
$$

Moreover, for any $T>0$ and $\tau>0$, the following estimates hold:

$$
\begin{aligned}
\operatorname{TV}\left(\rho^{\eta}(T, \cdot)\right) & \leq e^{C\left(\omega_{\eta}\right) T} T V\left(\rho_{0}\right), \\
\left\|\rho^{\eta}(T, \cdot)-\rho^{\eta}(T-\tau, \cdot)\right\|_{\mathbf{L}^{1}} & \leq \tau e^{C\left(\omega_{\eta}\right) T}\left(\left\|f^{\prime}\right\|\|v\|+J_{0}\|f\|\left\|v^{\prime}\right\|\right) T V\left(\rho_{0}\right),
\end{aligned}
$$

with $C\left(\omega_{\eta}\right):=\omega_{\eta}(0)\left(\left\|v^{\prime}\right\|\left(\left\|f^{\prime}\right\|\left\|\rho_{0}\right\|+2\|f\|\right)+\frac{7}{2} J_{0}\|f\|\left\|v^{\prime \prime}\right\|\right)$.

Under more regular assumptions, an estimate of the dependence of the solution with respect to the kernel function, the speed and the initial datum ca be proven, see [12]. Above, and in the sequel, we use the compact notation $\|\cdot\|$ for $\|\cdot\|_{\mathbf{L}^{\infty}}$.

Corollary 1 (Limit model as $\eta \rightarrow+\infty$ )

Let hypotheses $(\mathbf{H})$ hold and $\rho_{0} \in B V(\mathbb{R} ; I)$. As $\eta \rightarrow \infty$, the solution $\rho^{\eta}$ of (1), (5) converges in the $\mathbf{L}_{\mathbf{l o c}}^{\mathbf{1}}$-norm to the unique entropy weak solution of the classical Cauchy problem

$$
\begin{cases}\partial_{t} \rho+\partial_{x}(f(\rho) v(0))=0, & x \in \mathbb{R}, t>0 \\ \rho(0, x)=\rho_{0}(x), & x \in \mathbb{R} .\end{cases}
$$

In particular, we observe that $C\left(\omega_{\eta}\right) \rightarrow 0$ in (8a) and (8b), allowing to recover the classical estimates.

We observe that when the look-ahead distance $\eta \rightarrow \infty$, the non-local problem (1)-(5) becomes a classical transport equation (9). Besides the mathematical implications, Corollary 1 may give information on connected autonomous vehicle flow characteristics. Indeed, large kernel supports could account for the information range between 
connected autonomous vehicles. On the other hand, when the visibility $\eta \rightarrow 0$, the non-local problem (1)-(5) is again reduced formally to a local problem, but the singular local limit is more challenging from the mathematical point of view. The derivation of this limit was initially conjectured in [2] for the one-dimensional scalar case motivated by numerical evidence, later corroborated in [7]. See [15, 16, 22, 33] for more details regarding the analytical derivation of the singular local limit for non-local conservation laws.

\section{Non-local multi-class traffic flow models}

In this Section, we consider the following class of non-local systems of $M$ conservation laws in one space dimension, introduced in [11]:

$$
\partial_{t} \rho_{i}(t, x)+\partial_{x}\left(\rho_{i}(t, x) v_{i}\left(\left(r * \omega_{i}\right)(t, x)\right)\right)=0, \quad i=1, \ldots, M,
$$

where

$$
\begin{aligned}
& r(t, x):=\sum_{i=1}^{M} \rho_{i}(t, x), \quad v_{i}(\xi):=v_{i}^{\max } \psi(\xi), \\
& \left(r * \omega_{i}\right)(t, x):=\int_{x}^{x+\eta_{i}} r(t, y) \omega_{i}(y-x) d y,
\end{aligned}
$$

and we assume:

$$
\begin{aligned}
& \omega_{i} \in \mathbf{C}^{\mathbf{1}}\left(\left[0, \eta_{i}\right] ; \mathbb{R}^{+}\right), \eta_{i}>0 \text {, s.t. } \omega_{i}^{\prime} \leq 0, \int_{0}^{\eta_{i}} \omega_{i}(y) d y=J_{i} . \\
& W_{0}:=\max _{i=1, \ldots, M} \omega_{i}(0) .
\end{aligned}
$$

(H) $\quad v_{i}^{\max }$ are the maximal velocities $0<v_{1}^{\max } \leq v_{2}^{\max } \leq \ldots \leq v_{M}^{\max }$. $\psi: \mathbb{R}^{+} \rightarrow \mathbb{R}^{+}$is a smooth non-increasing function s.t.

$$
\psi(0)=1, \psi(r)=0 \text { for } r \geq 1 \text {. }
$$

For simplicity, we can consider the function $\psi(r)=\max \{1-r, 0\}$. We couple (10) with an initial datum

$$
\rho_{i}(0, x)=\rho_{i}^{0}(x), \quad i=1, \ldots, M .
$$

The model takes into account the distribution of heterogeneous drivers and vehicles characterized by their maximal speeds and look-ahead visibility in a traffic stream. It is a non-local generalization of the $n$-populations model for traffic flow described in [4] and it is a multi-class version of the one dimensional scalar conservation law with non-local flux proposed in [7], where $\rho_{i}$ is the density of vehicles belonging to the $i$-th class, $\eta_{i}$ is proportional to the look-ahead distance and $J_{i}$ is the interaction strength. The authors consider the following definition of weak solution because 
solutions to nonlinear conservation laws are in general discontinuous, even if the initial datum is smooth.

Definition 2 A function $\boldsymbol{\rho}=\left(\rho_{1}, \ldots, \rho_{M}\right) \in\left(\mathbf{L}^{1} \cap \mathbf{L}^{\infty}\right)\left(\left[0, T\left[\times \mathbb{R} ; \mathbb{R}^{M}\right), T>0\right.\right.$, is a weak solution of (10), (13) if

$$
\int_{0}^{T} \int_{-\infty}^{\infty}\left(\rho_{i} \partial_{t} \varphi+\rho_{i} v_{i}\left(r * \omega_{i}\right) \partial_{x} \varphi\right)(t, x) d x d t+\int_{-\infty}^{\infty} \rho_{i}^{0}(x) \varphi(0, x) d x=0,
$$

for all $\varphi \in \mathbf{C}_{\mathbf{c}}^{\mathbf{1}}(]-\infty, T[\times \mathbb{R} ; \mathbb{R}), i=1, \ldots, M$.

The main result in [11] is the proof of existence of weak solutions to (10), (13), locally in time.

Theorem 2 Let $\rho_{i}^{0}(x) \in\left(B V \cap \mathbf{L}^{\infty}\right)\left(\mathbb{R} ; \mathbb{R}^{+}\right)$, for $i=1, \ldots, M$, and assumptions $(\mathbf{H 1})$ - (H3) hold. Then the Cauchy problem (10), (13) admits a weak solution on $[0, T[\times \mathbb{R}$, for some $T>0$ sufficiently small.

\section{An upwind numerical scheme}

For the numerical simulations in Section 5, we consider the following conservative scheme for the model (10)-(13) introduced in [19] in the scalar case and generalized in [11] to the multi-class case. First of all, we extend $\omega_{i}(x)=0$ for $x>\eta_{i}$. For $j \in \mathbb{Z}$ and $n \in \mathbb{N}$, let $x_{j+1 / 2}=j \Delta x$ be the cell interfaces, $x_{j}=(j-1 / 2) \Delta x$ the cells centers and $t^{n}=n \Delta t$ the time mesh. We approximate the initial datum $\rho_{i}^{0}$ for $i=1, \ldots, M$ and the kernel as follows.

$\rho_{i, j}^{0}=\frac{1}{\Delta x} \int_{x_{j-1 / 2}}^{x_{j+1 / 2}} \rho_{i}^{0}(x) d x, \quad j \in \mathbb{Z} . \quad \omega_{i}^{k}:=\frac{1}{\Delta x} \int_{k \Delta x}^{(k+1) \Delta x} \omega_{i}(x) d x, \quad k \in \mathbb{N}$,

so that $\Delta x \sum_{k=0}^{+\infty} \omega_{i}^{k}=\int_{0}^{\eta_{i}} \omega_{i}(x) d x=J_{i}$. We underline that the sum is finite since $\omega_{i}^{k}=0$ for some $k \geq N_{i}$. Moreover, we set $r_{j+k}^{n}=\sum_{i=1}^{M} \rho_{i, j+k}^{n}$ for $k \in \mathbb{N}$ and

$$
V_{i, j}^{n}:=v_{i}^{\max } \psi\left(\Delta x \sum_{k=0}^{+\infty} \omega_{i}^{k} r_{j+k}^{n}\right), \quad i=1, \ldots, M, \quad j \in \mathbb{Z} .
$$

We obtain the following upwind scheme:

$$
\rho_{i, j}^{n+1}=\rho_{i, j}^{n}-\lambda\left(\rho_{i, j}^{n} V_{i, j+1}^{n}-\rho_{i, j-1}^{n} V_{i, j}^{n}\right),
$$

where we have set $\lambda=\frac{\Delta t}{\Delta x}$. 


\section{Numerical tests}

In this section we show some numerical simulations of the model (10)-(13) for $M=3$. The space step is $\Delta x=0.001$. We use the numerical scheme in Section 4 to approximate the solution of (10)-(13).

\subsection{Test 1: impact of connected autonomous trucks on a circular road}

We study the impact of connected autonomous trucks on road traffic performances on a circular road. For this reason, we consider the space interval $[-1,1]$ with periodic boundary conditions at $x= \pm 1$. In this case model (10)-(13) reads

$$
\left\{\begin{array}{l}
\partial_{t} \rho_{1}(t, x)+\partial_{x}\left(\rho_{1}(t, x) v_{1}^{\max } \psi\left(\left(r * \omega_{1}\right)(t, x)\right)\right)=0, \\
\partial_{t} \rho_{2}(t, x)+\partial_{x}\left(\rho_{2}(t, x) v_{2}^{\max } \psi\left(\left(r * \omega_{2}\right)(t, x)\right)\right)=0, \\
\partial_{t} \rho_{3}(t, x)+\partial_{x}\left(\rho_{3}(t, x) v_{3}^{\max } \psi\left(\left(r * \omega_{3}\right)(t, x)\right)\right)=0, \\
\rho_{1}(0, x)=\alpha(0.5+0.3 \sin (5 \pi x)), \\
\rho_{2}(0, x)=\beta(0.5+0.3 \sin (5 \pi x)), \\
\rho_{2}(0, x)=(1-\alpha-\beta)(0.5+0.3 \sin (5 \pi x)),
\end{array}\right.
$$

with

$$
\begin{array}{lrl}
\omega_{1}(x)=\frac{1}{\eta_{1}}, & \eta_{1}=0.3, \\
\omega_{2}(x)=\frac{2}{\eta_{2}}\left(1-\frac{x}{\eta_{2}}\right), & \eta_{2}=0.05, \\
\omega_{3}(x)=\frac{2}{\eta_{3}}\left(1-\frac{x}{\eta_{3}}\right), & \eta_{3}=0.1, \\
\psi(\xi)=\max \{1-\xi, 0\}, & \xi \geq 0, \\
v_{1}^{\text {max }}=0.8, \quad v_{2}^{\text {max }}=1.3, & v_{3}^{\text {max }}=0.8 . &
\end{array}
$$

Above $\rho_{1}$ represents the density of autonomous trucks, $\rho_{2}$ the density of humandriven cars and $\rho_{3}$ the density of human-driven trucks. $\alpha \in[0,1]$ is the penetration rate of autonomous trucks and $\beta \in[0,1]$ is the penetration rate of human-driven cars. Figure 1 displays the traffic dynamics in the case $\alpha=0.3$ and $\beta=0.5$, and Figure 2 displays the traffic dynamics in the case $\alpha=0$ and $\beta=0.5$ (no presence of autonomous trucks). We observe that oscillations are reduced if autonomous trucks are not present on the road, see Figure 3. 

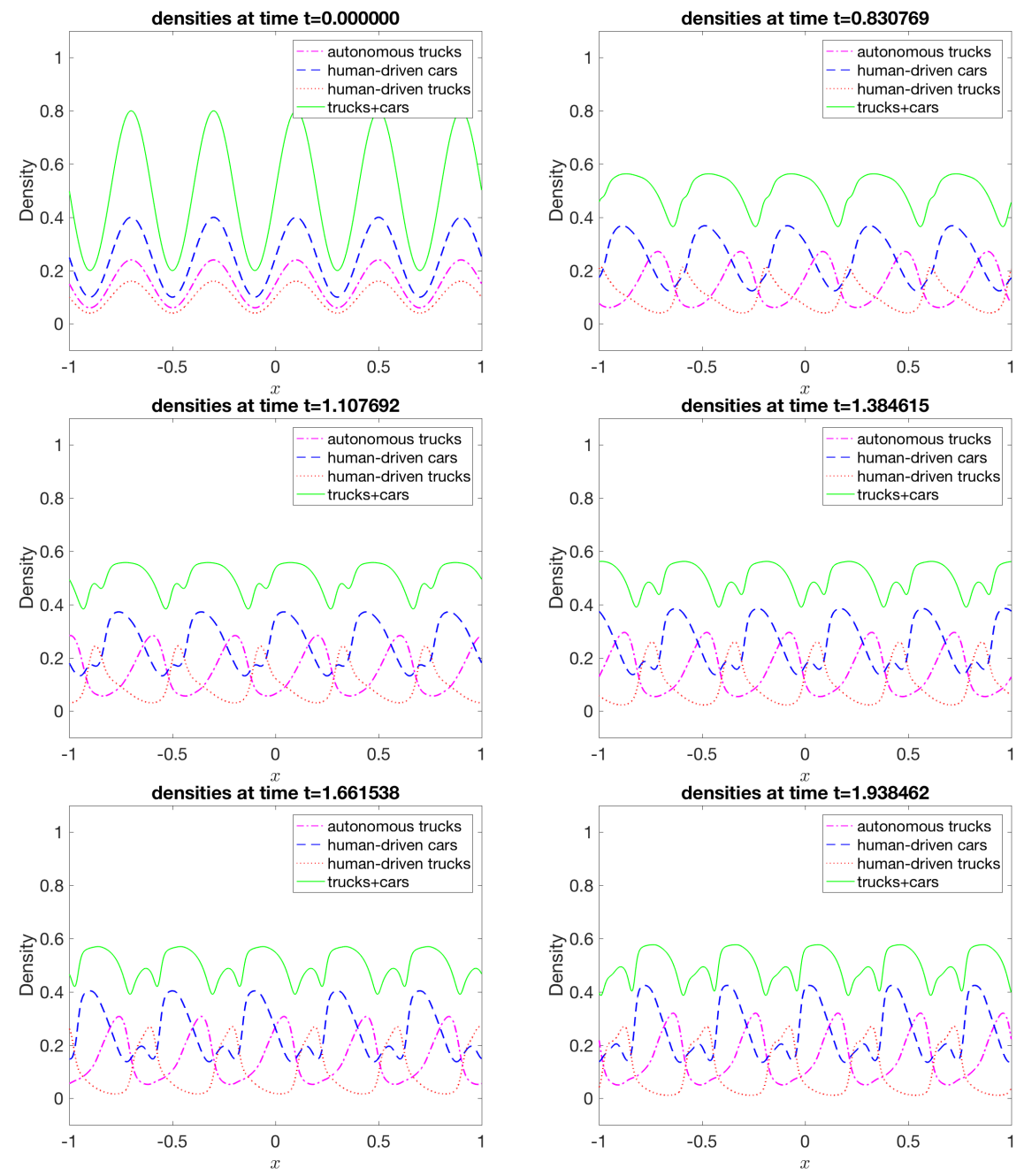

Fig. 1 Density profiles corresponding to the non-local problem (16) with $\alpha=0.3$ and $\beta=0.5$ at different times.

\subsection{Test 2: stretch of straight road with autonomous trucks}

In this test case, we propose a stretch of road with human-driven cars and trucks, and autonomous trucks. We consider the space domain $[-1,1]$, imposing absorbing boundary conditions at the boundaries $x= \pm 1$. The initial conditions for the equation (10) with $M=3$ are the following.

$\rho_{1}(0, x)=\alpha_{1} \chi_{[-0.6,-0.1](x)}$

$\omega_{1}(x)=\frac{1}{\eta_{1}}, \quad \eta_{1}=0.5, \quad v_{1}^{\max }=0.8$, 

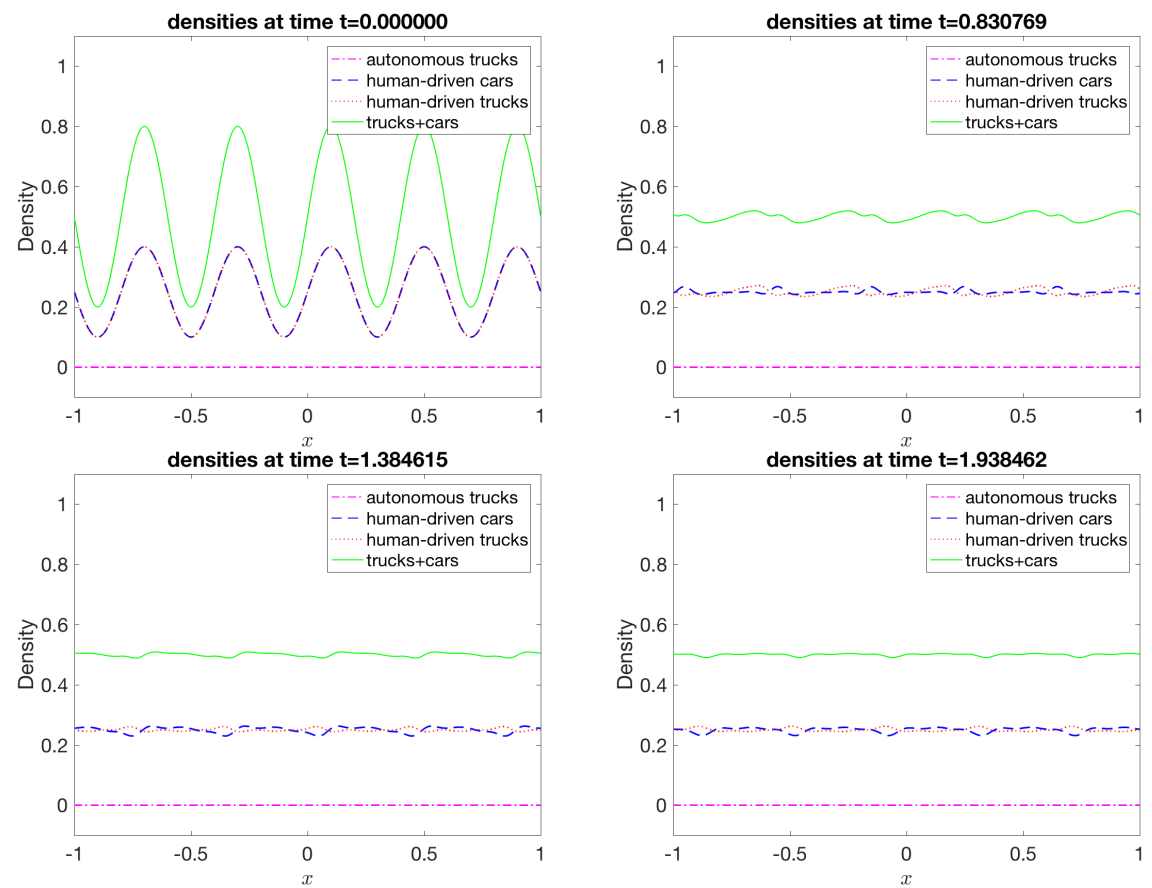

Fig. 2 Density profiles corresponding to the non-local problem (16) with $\alpha=0$ and $\beta=0.5$ at different times.
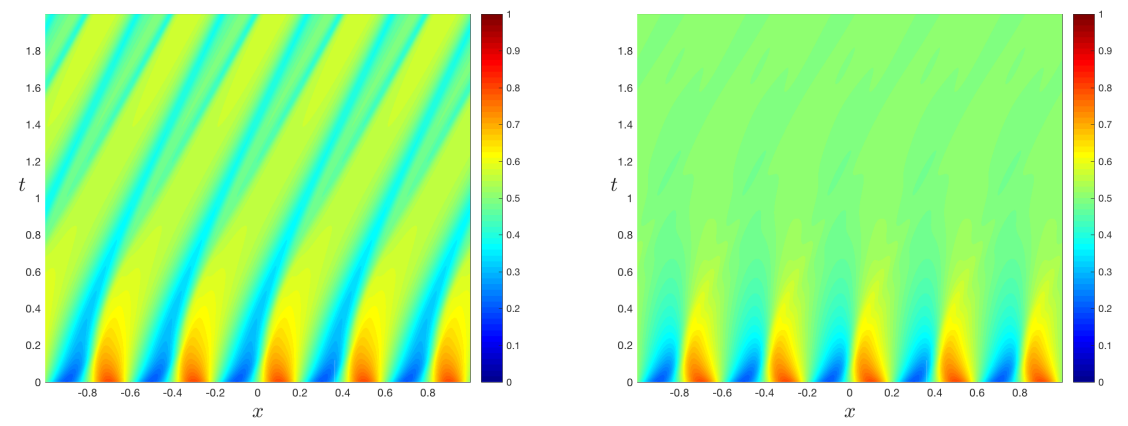

Fig. $3(t, x)$-plots of the total density $r(t, x)=\rho_{1}(t, x)+\rho_{2}(t, x)+\rho_{3}(t, x)$ computed with the upwind scheme (15), corresponding to different penetrations rate of autonomous trucks: (a) mixed autonomous/human-driven traffic, (b) fully human-driven traffic.

$$
\begin{aligned}
& \rho_{1}(0, x)=\left(1-\alpha_{1}\right) \chi_{[-0.6,-0.1](x)}, \quad \omega_{2}(x)=\frac{2}{\eta_{2}}\left(1-\frac{x}{\eta_{2}}\right), \quad \eta_{2}=0.1, \quad v_{2}^{\max }=0.8, \\
& \rho_{3}(0, x)=0.5 \chi_{[-0.9,-0.6](x),} \quad \omega_{3}(x)=\frac{2}{\eta_{3}}\left(1-\frac{x}{\eta_{3}}\right), \quad \eta_{3}=0.05, \quad v_{3}^{\max }=1.3,
\end{aligned}
$$

$\psi(\xi)=\max \{1-\xi, 0\}, \xi \geq 0$. 

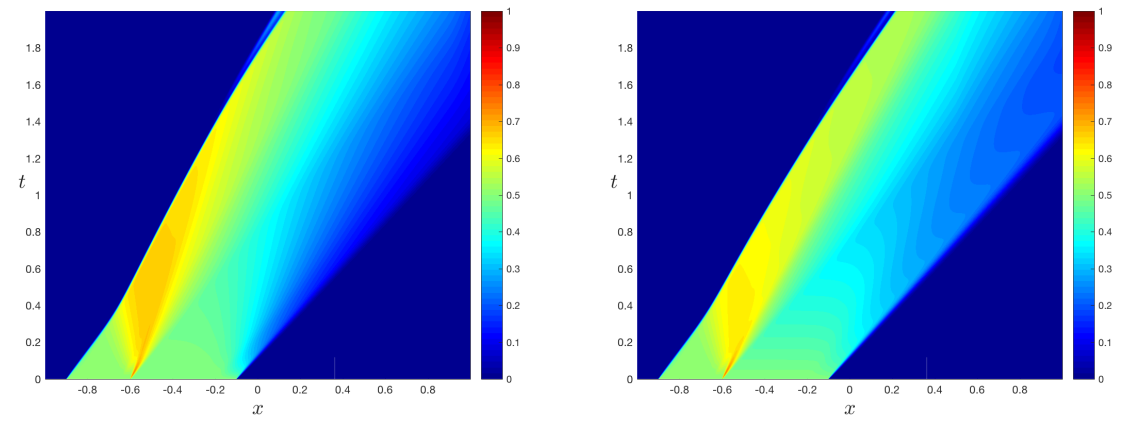

Fig. $4(t, x)$-plots of the total density $r(t, x)=\rho_{1}(t, x)+\rho_{2}(t, x)+\rho_{3}(t, x)$ computed with the upwind scheme (15), corresponding to different penetrations rate of autonomous trucks: (a) $\alpha_{1}=0.1$, (b) $\alpha_{1}=0.4$.

Above $\rho_{1}$ represents the density of autonomous trucks, $\rho_{2}$ the density of humandriven trucks and $\rho_{3}$ the density of human-driven cars. $\alpha_{1} \in[0,1]$ is the penetration rate of autonomous trucks. Figure 4 displays the traffic dynamics in the case $\alpha_{1}=0.1$ and $\alpha=0.4$. In this case, the presence of autonomous trucks reduces the vehicle densities during the overtaking phase, see Figure 4.

Acknowledgements The author wishes to thank Paola Goatin for her suggestions and valuable discussions.

\section{References}

1. A. Aggarwal, R. M. Colombo, and P. Goatin. Nonlocal systems of conservation laws in several space dimensions. SIAM J. Numer. Anal., 53(2):963-983, 2015.

2. P. Amorim, R. Colombo, and A. Teixeira. On the numerical integration of scalar nonlocal conservation laws. ESAIM M2AN, 49(1):19-37, 2015.

3. A. Aw and M. Rascle. Resurrection of "second order" models of traffic flow. SIAM J. Appl. Math., 60(3):916-938 (electronic), 2000.

4. S. Benzoni-Gavage and R. M. Colombo. An $n$-populations model for traffic flow. European J. Appl. Math., 14(5):587-612, 2003.

5. F. Berthelin and P. Goatin. Regularity results for the solutions of a non-local model of traffic flow. Discrete and Continuous Dynamical Systems A, 39(1078-0947 20196 3197):3197, 2019.

6. F. Betancourt, R. Bürger, K. H. Karlsen, and E. M. Tory. On nonlocal conservation laws modelling sedimentation. Nonlinearity, 24(3):855-885, 2011.

7. S. Blandin and P. Goatin. Well-posedness of a conservation law with non-local flux arising in traffic flow modeling. Numer. Math., 132(2):217-241, 2016.

8. C. Chalons, P. Goatin, and L. M. Villada. High-order numerical schemes for one-dimensional nonlocal conservation laws. SIAM J. Sci. Comput., 40(1):A288-A305, 2018. 
9. F. A. Chiarello, J. Friedrich, P. Goatin, S. Göttlich, and O. Kolb. A non-local traffic flow model for 1-to-1 junctions. European J. Appl. Math., 2019. to appear.

10. F. A. Chiarello and P. Goatin. Global entropy weak solutions for general non-local traffic flow models with anisotropic kernel. ESAIM Math. Model. Numer. Anal., 52(1):163-180, 2018.

11. F. A. Chiarello and P. Goatin. Non-local multi-class traffic flow models. Netw. Heterog. Media, 14(2):371 - 387, 2019.

12. F. A. Chiarello, P. Goatin, and E. Rossi. Stability estimates for non-local scalar conservation laws. Nonlinear Anal. Real World Appl., 45:668-687, 2019.

13. F. A. Chiarello, P. Goatin, and L. M. Villada. High-order Finite Volume WENO schemes for non-local multi-class traffic flow models. Proc. XVII International Conference on Hyperbolic Problems Theory, Numerics, Applications, 2019.

14. F. A. Chiarello, P. Goatin, and L. M. Villada. Lagrangian-antidiffusive remap schemes for non-local multi-class traffic flow models. Comput. Appl. Math., 2019. to appear.

15. M. Colombo, G. Crippa, M. Graffe, and L. V. Spinolo. Recent results on the singular local limit for nonlocal conservation laws. arXiv:1902.06970, 2019.

16. M. Colombo, G. Crippa, and L. V. Spinolo. On the singular local limit for conservation laws with nonlocal fluxes. arXiv e-prints, page arXiv:1710.04547, Oct 2017.

17. R. M. Colombo, M. Herty, and M. Mercier. Control of the continuity equation with a non local flow. ESAIM Control Optim. Calc. Var., 17(2):353-379, 2011.

18. J. Friedrich and O. Kolb. Maximum principle satisfying CWENO schemes for nonlocal conservation laws. SIAM J. Sci. Comput., 41(2):A973-A988, 2019.

19. J. Friedrich, O. Kolb, and S. Göttlich. A Godunov type scheme for a class of LWR traffic flow models with non-local flux. Netw. Heterog. Media, 13(4):531 - 547, 2018.

20. P. Goatin and S. Scialanga. Well-posedness and finite volume approximations of the LWR traffic flow model with non-local velocity. Netw. Heterog. Media, 11(1):107-121, 2016.

21. A. Keimer and L. Pflug. Existence, uniqueness and regularity results on nonlocal balance laws. J. Differential Equations, 263(7):4023-4069, 2017.

22. A. Keimer and L. Pflug. On approximation of local conservation laws by nonlocal conservation laws. J. Appl. Math. Anal. Appl., 475(2):1927 - 1955, 2019.

23. A. Keimer, L. Pflug, and M. Spinola. Existence, uniqueness and regularity of multi-dimensional nonlocal balance laws with damping. J. Math. Anal. Appl., 466(1):18 - 55, 2018.

24. S. N. Kružkov. First order quasilinear equations with several independent variables. Mat. Sb. (N.S.), 81 (123):228-255, 1970

25. A. Kurganov and A. Polizzi. Non-oscillatory central schemes for a traffic flow model with arrehenius look-ahead dynamics. Netw. Heterog. Media, 4(3):431-451, 2009.

26. D. Li and T. Li. Shock formation in a traffic flow model with Arrhenius look-ahead dynamics. Netw. Heterog. Media, 6(4):681-694, 2011.

27. M. J. Lighthill and G. B. Whitham. On kinematic waves. II. A theory of traffic flow on long crowded roads. Proc. Roy. Soc. London. Ser. A., 229:317-345, 1955.

28. H. Payne. Models of Freeway Traffic and Control. Simulation Councils, Incorporated, 1971.

29. P. I. Richards. Shock waves on the highway. Oper. Res., 4:42-51, 1956.

30. A. Sopasakis and M. A. Katsoulakis. Stochastic modeling and simulation of traffic flow: asymmetric single exclusion process with Arrhenius look-ahead dynamics. SIAM J. Appl. Math., 66(3):921-944 (electronic), 2006.

31. G. Whitham. Linear and nonlinear waves. Pure and applied mathematics. Wiley, 1974.

32. H. Zhang. A non-equilibrium traffic model devoid of gas-like behavior. Transportation Research Part B: Methodological, 36(3):275 - 290, 2002.

33. K. Zumbrun. On a nonlocal dispersive equation modeling particle suspensions. Quart. Appl. Math., 57(3):573-600, 1999. 